\title{
Dielectric discontinuity effects on the adsorption of a linear polyelectrolyte at the surface of a neutral nanoparticle
}

\author{
Marianne Seijo, ${ }^{1}$ Martin Pohl, ${ }^{2}$ Serge Ulrich, ${ }^{1}$ and Serge Stoll ${ }^{1, a)}$ \\ ${ }^{1}$ Analytical and Biophysical Environmental Chemistry (CABE), Institute F.A. Forel, University of Geneva, \\ 10 route de Suisse, Case Postale 416, CH-1290 Versoix, Switzerland \\ ${ }^{2}$ Department of Nuclear and Corpuscular Physics, University of Geneva, 24 quai E. Ansermet, \\ CH-1211 Geneva 4, Switzerland
}

(Received 23 July 2009; accepted 30 September 2009; published online 3 November 2009)

\begin{abstract}
The formation of complexes between nanoparticles and polyelectrolytes is a key process for the control of the reactivity of manufactured nanoparticles and rational design of core shell nanostructures. In this work, we investigate the influence of the nanoparticle dielectric constant on the adsorption of a linear charged polymer (polyelectrolyte) at the surface of a neutral nanoparticle. The polyelectrolyte linear charge density, as well as the image charges in the nanoparticle due to the dielectric discontinuity, is taken into account. Monte Carlo simulations are used to predict the adsorption/desorption limits and system properties. Effects of the nanoparticle size and polyelectrolyte length are also investigated. The polyelectrolyte is found adsorbed on the nanoparticle when the dielectric constant of the nanoparticle is greater than the dielectric constant of the medium. Attractive interactions induced by the presence of opposite sign image charges are found strong enough to adsorb the polyelectrolyte showing that the reaction field contribution has to be considered. The affinity between the polyelectrolyte and the nanoparticle is found to increase in magnitude by increasing the nanoparticle size and dielectric constant. The reaction field magnitude is also found to depend in a nonlinear way from the polyelectrolyte length. (c) 2009 American Institute of Physics. [doi:10.1063/1.3251767]
\end{abstract}

\section{INTRODUCTION}

It is well known that the properties of materials change as their size approaches the nanoscale. In particular due to their specific nanometric sizes, engineered nanoparticles (NPs) as well as natural NPs have high specific surface areas, which make them potentially very reactive, catalytic, and diffusive. ${ }^{1}$ For the control of the NP reactivity, the surfaces of engineered NPs are frequently modified (e.g., functionalized), coated with polymers to prevent aggregation, or alter their properties. For that purpose polyelectrolyte $(\mathrm{PE})$ chains (charged polymers) are usually used for the formation of PE and NP complexes. ${ }^{2,3}$ Coating and functionalization have also implications for mobility and biological uptake when engineered NPs are released in environmental systems. While some inorganic polymeric coatings are stable, some organic coatings are biodegradable and will ultimately disappear to release core particles and expose their surface to the environment and organisms. Thus, a better understanding of the key factors controlling the coating and complexation processes between PEs and NPs at the nanoscale level is therefore essential for the rational use of engineered NPs and understanding their possible impact on living organisms and ecosystems. ${ }^{4}$

The complexation of PEs on various substrates has been investigated by a range of experimental methods, theoretical models, and computer simulations. ${ }^{5-7}$ A series of contribu-

\footnotetext{
a) Author to whom correspondence should be addressed. Electronic mail: serge.stoll@unige.ch.
}

tions from Dubin and co-workers ${ }^{6,8-12}$ was made on the adsorption of PEs to spherical nanometric size micelles, dendrimers, and proteins using both experimental and computer simulations. These contributions pointed out the importance of the NP surface charge density and revealed that for PEs of high linear charge density, complexation was electrostatically driven. Adsorption processes of DNA rigid fragment on spherical latex particles were investigated by Elaissari and co-workers ${ }^{13-15}$ and demonstrated the major role of electrostatic forces in the adsorption processes. They found that the adsorption amount and surface conformation of oligonucleotides were strongly dependent on the $\mathrm{pH}$ and ionic strength of the medium and the charge density of the latex particles. The contribution of hydrophobic forces was also evidenced using various adsorption isotherms at various $\mathrm{pHs}$ and extrapolating these results to zero surface charge density and zero zeta potential of the latex particles. Grant and Walker ${ }^{16,17}$ examined the relationship between polymer surface conformation and latex stability using single-stranded DNA as a model polymer. They also showed the importance of electrostatic forces on the polymer conformation and flexibility at the surface of the latex particles. Manning ${ }^{18} \mathrm{dem}-$ onstrated that for a given PE persistence length, the curvature of charged spherical NPs imposed stability limits of the complexes owing to the fact that it was more difficult to adsorb a PE to a smaller NP than a larger one.

However there is one important interfacial property which is generally not considered in the formation of complexes between NPs and PEs or when describing the electrical behavior of charged macroions: The dielectric disconti- 
nuity. When a dielectric discontinuity between two media, with relative dielectric constants $\varepsilon_{1}$ and $\varepsilon_{2}$, is considered, a charge $\mathrm{q}$ in medium 1 at a distance $d$ from the dielectric discontinuity is expected to interact with the interface because of the presence of a reaction field issue from the polarization of both media. ${ }^{19}$ This results in the formation of an "image" charge $\mathrm{q}_{0}$ located at the symmetric positions $2 d$ of q. If $\varepsilon_{1}<\varepsilon_{2}$, the resulting reaction field force is attractive. If $\varepsilon_{1}>\varepsilon_{2}$, the resulting reaction field force is repulsive, whereas if $\varepsilon_{1}=\varepsilon_{2}$ there is no reaction field. It should be noted that when the number of charges in the system is large due to the many-body nature of the problem and the long range influence of the electrostatic interactions, an exact calculation is generally not accessible. Then, numerical simulations need to be performed. Linse ${ }^{20}$ recently presented a united description of the electrostatics of an arbitrary number of electrostatic multipoles, each localized in a spherical cavity possessing a dielectric permittivity different from that of the surrounding. Netz ${ }^{21}$ investigated the effects of the dielectric constant discontinuity of an uncharged surface on the adsorption process of a weak rod like PE composed of 20 monomers. Results were discussed as a function of the distance of the $\mathrm{PE}$ to the surface by considering high (metallic compound) and low (globular protein) dielectric constants. At high dielectric constant (fixed to $\infty$ ), the effective charge was found to increase and the PE was attracted to the surface, while at low dielectric constant of the surface (fixed to 0), repulsion from the surface was found. The adsorption behavior of a PE chain near high dielectric constant surface materials was therefore predicted to be dominated by imagecharge attraction, leading to a logarithmic (and thus very weak) dependence on the screening length and a qualitative agreement with experimental observations. ${ }^{22}$ Dobrynin and Rubinstein $^{23}$ studied the adsorption of a flexible PE at a flat surface with a dielectric discontinuity including the effect of van der Waals interactions. Considering an uncharged and low dielectric constant surface, it was shown that the repulsion of the PE from its image charges can be safely neglected only if the latter is much smaller than the van der Waals short-range interactions. Cheng and $\mathrm{Lai}^{24}$ studied the behavior of a PE adsorbed on a charged and high-dielectric constant surface. They found that the PE image charge attraction was able to overcome the repulsive interactions with the surface, hence resulting in $\mathrm{PE}$ adsorption. Messina ${ }^{25,26}$ performed Monte Carlo simulations to investigate the effect of the dielectric discontinuity on the adsorption of strongly charged PEs at an oppositely charged planar surface having a lower dielectric constant than the solvent. Simulations indicated that image charge formation was considerably reducing the degree of PE adsorption preventing surface charge inversion by the adsorbed PEs.

Nonetheless most of these studies were performed by considering planar interfaces without considering curvature effects. In the present contribution, we investigate in a systematic way the adsorption (complexation) process between a charged PE and uncharged spherical NPs to quantify the influence and reaction field magnitude originating from the image surface charges appearing at the dielectric interface. Curvature effects are investigated by adjusting the NP sizes

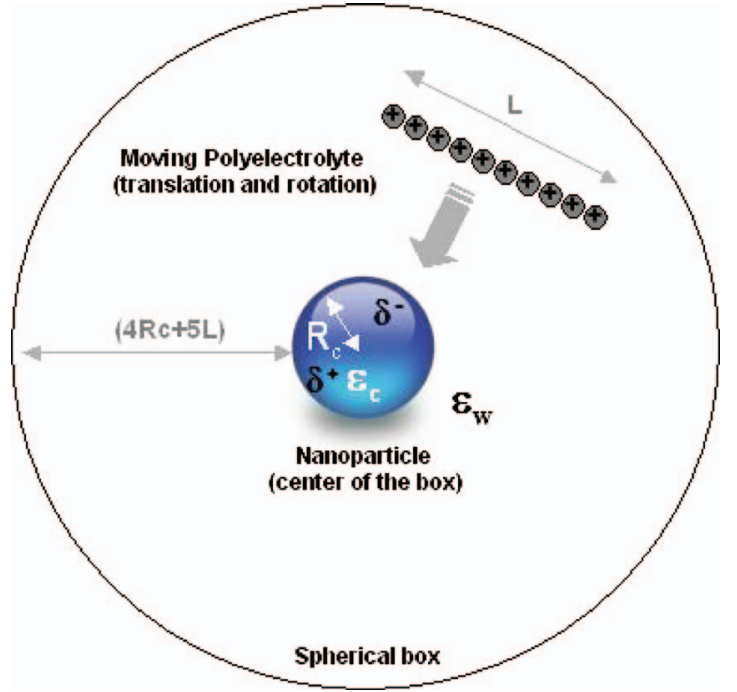

FIG. 1. Coarse grained model used to study the adsorption of a PE chain at the surface of a neutral NP in the presence of dielectric discontinuities. The spherical NP is fixed at the center of a spherical cell. A rigid and strong PE is placed inside the cell and submitted to elementary movements. A Monte Carlo simulation algorithm is then used to investigate the complex formation conditions by adjusting the dielectric constant of the NP and relative size between the PE and NP. In theoretical modeling the surface-induced charge regulation of the polymer has to be included.

and PE length. Special attention is made to the reaction field part contributions due to the dielectric discontinuity between the NPs and the solvent. For that purpose the dielectric constant of the NPs was systematically adjusted to mimic the behavior of several materials from proteins to metallic compounds.

\section{THEORETICAL APPROACH AND MODEL}

\section{A. Model description}

A rigid PE and a spherical NP are represented at a coarse grained level and off-lattice three-dimensional simulations are considered. The solvent is treated here as a dielectric continuum medium with a relative dielectric permittivity $\varepsilon_{\mathrm{W}}$ taken to that of water at $298 \mathrm{~K}$, i.e., 78.54. The PE chain is represented by $\mathrm{N}_{\mathrm{m}}$ jointed hard spheres (or monomers) to take into account the excluded volume effect. We assume that the intrinsic dielectric constant of the PE is equal to that of water. The PE is strictly linear and rigid so as to represent a rodlike conformation. Each PE monomer carries a negative charge at its center. As a result strong PEs are considered in this study. To avoid the Manning ${ }^{27}$ counterion condensation regime, the monomer radius is set to $1_{B} / 2.0=3.57 \AA$, where $\mathrm{l}_{\mathrm{B}}$ represents the Bjerrum length at $298 \mathrm{~K}$. The NP is represented as an impenetrable and solvent excluded sphere with a variable radius $R_{c}$ and dielectric constant $\varepsilon_{c}$, which can be adjusted so as to be different from the dielectric constant of the surrounding water $\varepsilon_{\mathrm{w}}$ (Fig. 1). In our simulations, we consider that the PEs remain rigid during the adsorption process for several reasons: (i) PE linear charge density, (ii) high electrostatic PE persistence length, and (iii) important intrinsic persistence length of many natural polysaccharides. From a prospective point of view, it should be very interesting to 
TABLE I. Geometrical maximum number of monomers (radius=3.57 $\AA$ ) adsorbed at the surface of a NP with a radius $R_{c}$ according to the adsorption criteria defined in the text. If the geometrical maximum number of monomers adsorbed is higher than the PE monomer number, $\max \left(\mathrm{N}_{\mathrm{ads}}\right)$ is then equal to the total number of monomers in the polymer.

\begin{tabular}{llrrrr}
\hline \hline $\mathrm{R}_{\mathrm{c}}(\mathrm{nm})$ & 3.57 & 35.7 & 150.0 & 250.0 & 357.0 \\
Maximum number of monomers & 4.6 & 14.0 & 28.8 & 37.2 & 44.5 \\
in the NP adsorption layer & & & & & \\
\hline \hline
\end{tabular}

introduce some polymer flexibility within the model, hence allowing the PE chains to be completely adsorbed at the NP surface.

In our simulations, the total electrostatic interaction potential between the NP and the PE includes both the direct Coulomb and reaction field contributions. The reaction field is directly issued from the dielectric discontinuity through the solid-NP/liquid interface. The interaction potential calculation is based on the classical approach derived by Böttcher. ${ }^{28}$ Given the elementary charge e $\left(1.63 \times 10^{-19} \mathrm{C}\right)$ and the vacuum permittivity $\varepsilon_{0}\left(8.85 \times 10^{-12} \mathrm{C} \mathrm{V}^{-1} \mathrm{~m}^{-1}\right), \mathrm{P}_{\mathrm{n}}^{0}$ the ordinary Legendre polynomial of order $n$ and $n_{\max }$ the maximum number of terms included in the sum, two ionized monomers $i$ and $j$ of charge $z_{i}$ and $z_{j}$ on the PE, located at a radial position $r_{i}$ and $r_{j}$ from the center of the cell, separated by a distance $\mathrm{r}_{\mathrm{ij}}$ with an angle $\theta_{\mathrm{ij}}\left[\right.$ with $\cos \left(\theta_{\mathrm{ij}}\right)=1-\mathrm{r}_{\mathrm{ij}}^{2} / 2 \mathrm{r}_{\mathrm{i}} \mathrm{r}_{\mathrm{j}}$ ], contribute to a total electrostatic potential energy (in $\mathrm{k}_{\mathrm{B}} \mathrm{T}$ units) given by

$$
\begin{aligned}
\mathrm{E}_{\text {mono-mono }}(\mathrm{i}, \mathrm{j})= & \frac{\mathrm{z}_{\mathrm{i}} \mathrm{Z}_{\mathrm{j}} \mathrm{e}^{2}}{4 \pi \varepsilon_{0} \varepsilon_{\mathrm{w}}} \frac{1}{\mathrm{k}_{\mathrm{B}} \mathrm{T}}\left(\frac{1}{\mathrm{r}_{\mathrm{ij}}}\right. \\
& \left.+\frac{1}{\mathrm{R}_{\mathrm{c}}} \sum_{\mathrm{n}=1}^{\mathrm{n}_{\max }} \frac{\left(\varepsilon_{\mathrm{w}}-\varepsilon_{\mathrm{c}}\right) \mathrm{P}_{\mathrm{n}}^{0}\left(\cos \theta_{\mathrm{ij}}\right)}{\left(\varepsilon_{\mathrm{c}}+\varepsilon_{\mathrm{w}}(1+1 / \mathrm{n})\right)}\left(\frac{\mathrm{R}_{\mathrm{c}}^{2}}{\mathrm{r}_{\mathrm{i}} \mathrm{r}_{\mathrm{j}}}\right)^{\mathrm{n}+1}\right) .
\end{aligned}
$$

The first term of the summation corresponds to the direct Coulomb contribution $\mathrm{E}_{\text {Coulomb }}$, whereas the second term concerns the reaction field contribution $\mathrm{E}_{\mathrm{RF}}$. Hard-core repulsions between the NP and the PE monomers result to an excluded volume interaction given by

$$
\mathrm{E}_{\text {exc NP-PE }}(\mathrm{i})=\left\{\begin{array}{ll}
\infty & \text { if } \mathrm{r}_{\mathrm{i}}<\left(\mathrm{R}_{\mathrm{c}}+\mathrm{R}_{\mathrm{m}}\right) \\
0 & \text { otherwise }
\end{array} .\right.
$$

On the other hand, the excluded volume between the PE and the cell is given by

$$
E_{\text {exc PE-cell }}(i)=\left\{\begin{array}{ll}
\infty & \text { if } r_{i}>5\left(R_{c}+L\right) \\
0 & \text { otherwise }
\end{array},\right.
$$

where $L=\left(2 \times R_{m} \times N_{m}\right)$ represents the contour length of the $\mathrm{PE}$ and $\mathrm{N}_{\mathrm{m}}$ the number of monomers. by

The total energy $E_{\text {tot }}$ of the system is subsequently given

$$
\begin{aligned}
\mathrm{E}_{\text {tot }}= & \sum_{\mathrm{i}=1}^{\mathrm{N}_{\mathrm{m}}}\left(\mathrm{E}_{\text {exc }} \text { PE-NP }(i)+\mathrm{E}_{\text {exc PE-cell }}(\mathrm{i})\right) \\
& +\sum_{\mathrm{i}=1}^{\left(\mathrm{N}_{\mathrm{m}}-1\right)} \sum_{\mathrm{j}>\mathrm{i}}^{\mathrm{N}_{\mathrm{m}}}\left(\mathrm{E}_{\text {mono-mono }}(\mathrm{i}, \mathrm{j})\right) .
\end{aligned}
$$

\section{B. Monte Carlo simulation method}

To explore the system configuration space, Monte Carlo Metropolis $^{29}$ simulations are made in the canonical ensemble. One NP is placed at the center of a large threedimensional spherical reflecting box of radius $R_{\text {cell }}$ equal to $5\left(\mathrm{R}_{\mathrm{c}}+\mathrm{L}\right)$. Once the PE is introduced in the cell, elementary and random movements (translation and rotation) are allowed. Each movement is accepted or not by considering the Metropolis selection criterion. The acceptance criterion is sensitive to the value of the total energy variation $\Delta \mathrm{E}$. If the change in the total energy $\Delta \mathrm{E}$ resulting from the movement is negative, then the system has a lower energy and the move is selected. If $\Delta \mathrm{E}$ is positive, a Boltzmann factor given by $\mathrm{p}=\exp \left(-\Delta \mathrm{E} / \mathrm{k}_{\mathrm{B}} \mathrm{T}\right)$ is calculated and compared with a random number $\mathrm{z}$ (with $0 \leq \mathrm{z} \leq 1$ ). If $\mathrm{z} \leq \mathrm{p}$, the movement is accepted. If $\mathrm{z}>\mathrm{p}$, the movement is rejected and the previous $\mathrm{PE}$ position is considered as a new state in calculating ensemble averages. After an equilibration period equal to $5 \times 10^{5}$ cycles, system properties are calculated during $2 \times 10^{6}$ cycles. During this production period, results are recorded every 500 cycles.

\section{RESULTS AND DISCUSSION}

In order to characterize the PE adsorption and its affinity with the NP surface, a parameter $\tau$ is defined according to

$$
\tau(\%)=\frac{100 \times\left\langle\mathrm{N}_{\mathrm{ads}}\right\rangle}{\max \left(\mathrm{N}_{\mathrm{ads}}\right)}
$$

where $\left\langle\mathrm{N}_{\mathrm{ads}}\right\rangle$ represents the average number of monomers in the adsorption layer of the NP (set to three times the monomer radius) and $\max \left(\mathrm{N}_{\mathrm{ads}}\right)$ the maximum number of possible adsorbed monomers in the NP adsorption layer. $\left\langle\mathrm{N}_{\mathrm{ads}}\right\rangle$ is obtained from simulation results when statistical convergence is observed (during the production period). The geometrical maximum number of monomers in the adsorption layer $\max \left(\mathrm{N}_{\mathrm{ads}}\right)$, which is a function of the NP curvature, is given in Table I for different NP radii, $\mathrm{R}_{\mathrm{c}}$.

According to the parameter defined here to discuss the PE-NP adsorption affinity, the PE is never adsorbed when $\tau$ is equal to $0 \%$. In contrast, when $\tau$ is close to $100 \%$, the PE has a strong affinity with the NP. Intermediate values denote that only a fraction of the PE is adsorbed and the adsorption process is reversible. Hence the PE is continuously adsorbed and desorbed from the NP but remains in its vicinity. 


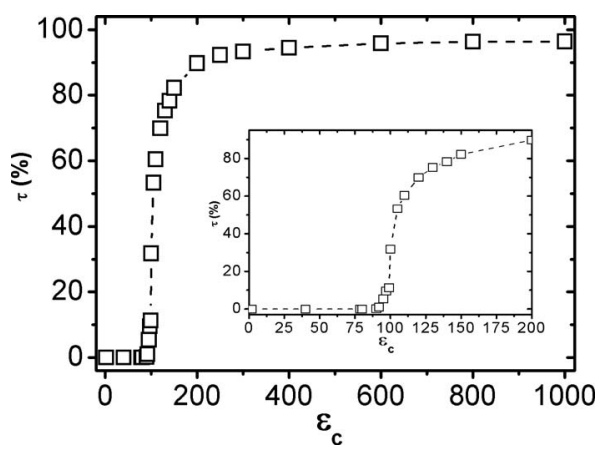

FIG. 2. Variation in the PE-NP affinity $\tau(\%)$ as a function of the NP dielectric constant. The NP radius here is equal to $R_{c}=35.7 \mathrm{~nm}$ and the number of monomers in the PE, $\mathrm{N}_{\mathrm{m}}$, is equal to 60 . $\mathrm{PE}$ adsorption is rapidly promoted by increasing the NP dielectric constant above the water dielectric constant (at $\varepsilon_{\mathrm{c}} \simeq 100$ ), then a plateau value is achieved.

\section{A. Dielectric constant effects on the complex formation}

The effect of the dielectric discontinuity between the NP and the solvent on the PE adsorption is first explored. The radius of the NP has been set to a constant value equal to $35.7 \mathrm{~nm}$. Several NP dielectric constants are used here from high $\left(\varepsilon_{\mathrm{c}}=1000\right.$, representative of some metals $)$ to low $\varepsilon_{\mathrm{c}}$ values $\left(\varepsilon_{\mathrm{c}}=2\right.$, values found in proteins) and intermediate values $\left(\varepsilon_{\mathrm{c}}=140,110,40\right.$ and $\left.\varepsilon_{\mathrm{c}}=\varepsilon_{\mathrm{w}}=78.54\right)$. As observed in Fig. 2, when $\varepsilon_{\mathrm{c}} \leq \varepsilon_{\mathrm{w}}$, no adsorption is found between the NP and the $\mathrm{PE}$ due to the presence of the repulsive interactions induced by the reaction field and presence of image charges having the same sign than the PE. No adsorption is observed when $\varepsilon_{\mathrm{c}}=\varepsilon_{\mathrm{w}}$, since the reaction field contribution is null. On the other hand, when $\varepsilon_{\mathrm{c}}>\varepsilon_{\mathrm{w}}$, due to the formation of image charges of opposite signs within the NP, and attractive interactions induced by the reaction field, PE adsorption becomes more and more efficient with increasing the NP dielectric constant. A rapid increase is observed when $\varepsilon_{\mathrm{c}}=100$ then a plateau value close to $97 \%$ is achieved for dielectric constant values larger than 200 .

\section{B. NP size influences}

The effect of the NP size and curvature is explored by adjusting the NP radius to values between 3.57 and $357 \mathrm{~nm}$. The NP dielectric constant effect is also considered by performing calculations at various dielectric constants. As observed in Fig. 3, the dielectric discontinuity effect, which is obtained when $\varepsilon_{\mathrm{c}}>\varepsilon_{\mathrm{w}}$, is significantly controlled by the NP size. When the NP radius increases, the affinity between the NP and the PE also increases due to an increase in the number of monomers in the adsorption layer (geometrical effect) and increase in the reaction field contribution to the energy. Thus increasing the NP size promotes the PE affinity and NP-PE complex formation when $\varepsilon_{\mathrm{c}}>\varepsilon_{\mathrm{w}}$.

In Fig. 4 the reaction field energy is plotted as a function of the radial distance $d$ between the central part of the PE and the NP surface for different NP radii and NP dielectric constant values. As expected, the reaction field is found positive when $\varepsilon_{\mathrm{c}}<\varepsilon_{\mathrm{w}}$, whereas when $\varepsilon_{\mathrm{c}}>\varepsilon_{\mathrm{w}}$, negative values are obtained. It should be noted that when the NP is small (a few nanometers), the reaction field intensity and range of inter-

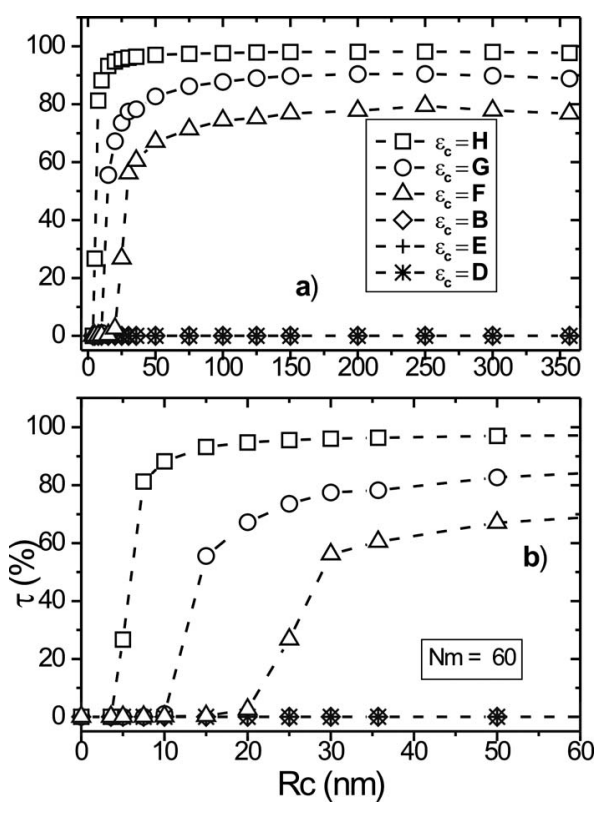

FIG. 3. Variation in the PE-NP affinity $\tau(\%)$ as a function of the NP dielectric constant and NP size. Adsorption is found promoted by both increasing the NP radius $\mathrm{R}_{\mathrm{c}}$ and dielectric NP constant $\varepsilon_{\mathrm{c}}\left(\right.$ when $\left.\varepsilon_{\mathrm{c}}>\varepsilon_{\mathrm{w}}\right)$.

action between the PE and NP are relatively low. By increasing the NP radius both the range (in comparison to the NP size) and the reaction field intensity increase significantly. For example for a NP having a radius equal to $150 \mathrm{~nm}$ a significant attractive interaction is found up to $45 \mathrm{~nm}$. It should be noted that at short separation distances attractive interaction energies are significant and non-negligible with regard to conventional electrostatic Coulomb interactions (for e.g., existing between charged NPs).

\section{PE chain length effects}

The influence of the PE chain length on the PE adsorption process is presented in Fig. 5 at different NP dielectric constants. Several chain lengths have been considered with 60,80 , and 100 monomers. It is shown that a moderate increase in the PE-NP affinity is achieved with increasing the PE length, in particular when the NP dielectric constant decreases to the water dielectric constant. Both the NP radius and the PE length are shown to promote the adsorption process. Here the plateau value for the affinity parameter is clearly found to decrease with the dielectric constant of the NP. Adjusting systematically the PE length and NP size at $\varepsilon_{\mathrm{c}}=1000$, we calculated the total reaction field interaction as a function of the total PE monomer number $\mathrm{N}_{\mathrm{m}}$. As shown in Fig. 6, a minimum value for the reaction field intensity is found for a particular PE size. Such a value, $\mathrm{N}_{\min }$, corresponds to the best conditions for the formation of a stable complex between the PE and the NP by considering the dielectric discontinuity, NP size, and PE length. As shown in Figs. 6(a) and 6(b), such a minimum value, $\mathrm{N}_{\min }$, and corresponding reaction field intensity are found to depend on the NP size. Increasing the NP size results in an increase in the $\mathrm{N}_{\min }$ value. By adjusting the $\mathrm{R}_{\mathrm{m}}$ value we also found that the minimum of the total reaction field was observed when $\mathrm{N}_{\min } \simeq 2.4 \mathrm{R}_{\mathrm{c}} / \mathrm{R}_{\mathrm{m}}$. In order to explain the presence of such a 

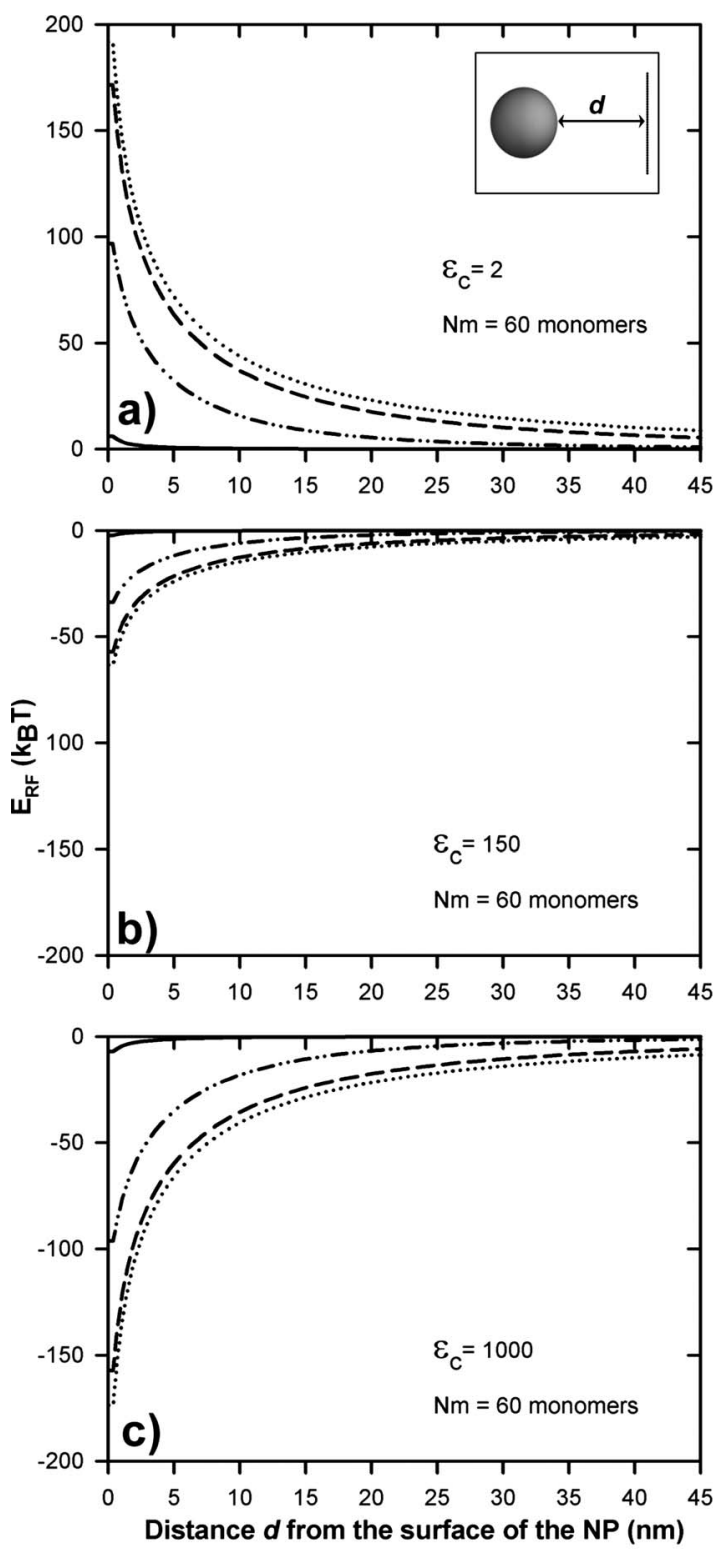

\begin{tabular}{llll} 
& $\mathrm{Rc}=3.57 \mathrm{~nm}$ & $-\cdots--$ & $\mathrm{Rc}=150 \mathrm{~nm}$ \\
\hdashline$\cdots \cdots-.$. & $\mathrm{Rc}=35.7 \mathrm{~nm} \quad \ldots \ldots \ldots \ldots .$. & $\mathrm{Rc}=357 \mathrm{~nm}$
\end{tabular}

FIG. 4. Reaction field values (in $\mathrm{k}_{\mathrm{B}} \mathrm{T}$ units) resulting from the PE-image charge interactions as a function of the distance $d$ from the NP surface (see the inset). The solvent (water) has a dielectric constant $\varepsilon_{\mathrm{w}}=78.54$. The NP has a dielectric constant of (a) $\varepsilon_{\mathrm{c}}=2$, (b) $\varepsilon_{\mathrm{c}}=150$, and (c) $\varepsilon_{\mathrm{c}}=1000$. The NP radius is set to $3.57,35.7,150$, and $357 \mathrm{~nm}$. Long range effects are observed in comparison to the NP sizes.

minimum, the individual contribution of each monomer to the reaction field energy was calculated and plotted in Figs. $7(\mathrm{a})$ and $7(\mathrm{~b})$ as a function of the monomer position. A PE with 201 monomers [Fig. 7(a)] is considered and it is assumed that the PE position is tangential to the NP and adsorbed by its central part. As observed, in a region close to the PE-NP contact, the reaction field is fully attractive. This region concerns a well-defined number of monomers whose value is given by the $\mathrm{N}_{\min }$ value previously discussed. Out of this region, the interactions between the rest of the monomers and the NP are slightly repulsive (positive $\mathrm{E}_{\mathrm{RF}}$ values are obtained) and decrease to zero by increasing the monomer position. In Fig. 7(b), a short PE with 11 monomers is
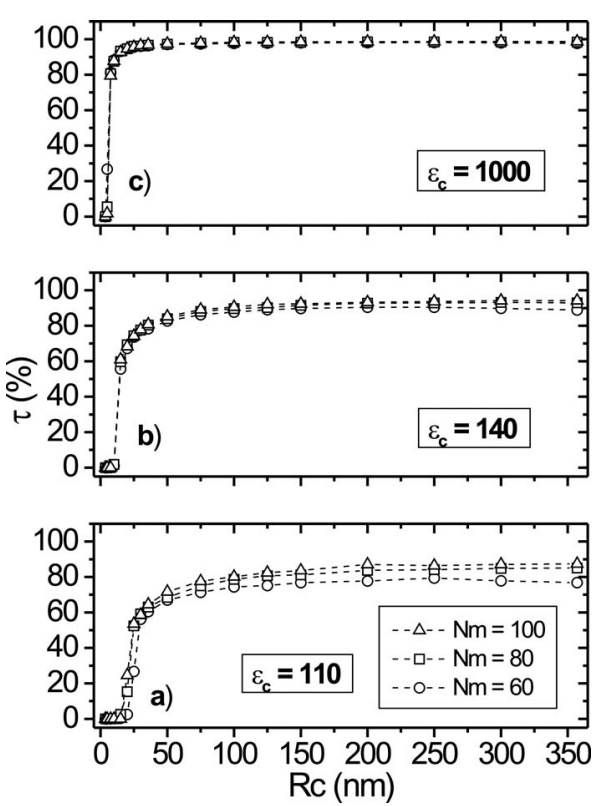

FIG. 5. Effects of the PE chain length, dielectric constant $\varepsilon_{c}$, and NP radius on the NP-PE affinity. Three NP dielectric constants are considered: (a) $\varepsilon_{\mathrm{c}}$ $=1000$, (b) $\varepsilon_{\mathrm{c}}=140$, and (c) $\varepsilon_{\mathrm{c}}=110$. It is found that the NP-PE affinity is promoted by increasing the $\mathrm{PE}$ length, dielectric NP constant, and to a large extent by increasing the NP radius.

considered. In that case all the individual monomer contributions to the reaction field are negative since $\mathrm{N}_{\mathrm{m}}<\mathrm{N}_{\min }$. This is an important result showing that a complex distribution of positive and negative image charges is achieved within the $\mathrm{NP}$ at a critical PE length.

\section{CONCLUSIONS}

The dielectric discontinuity effects on the complexation between a rigid PE and a neutral NP have been explored. The
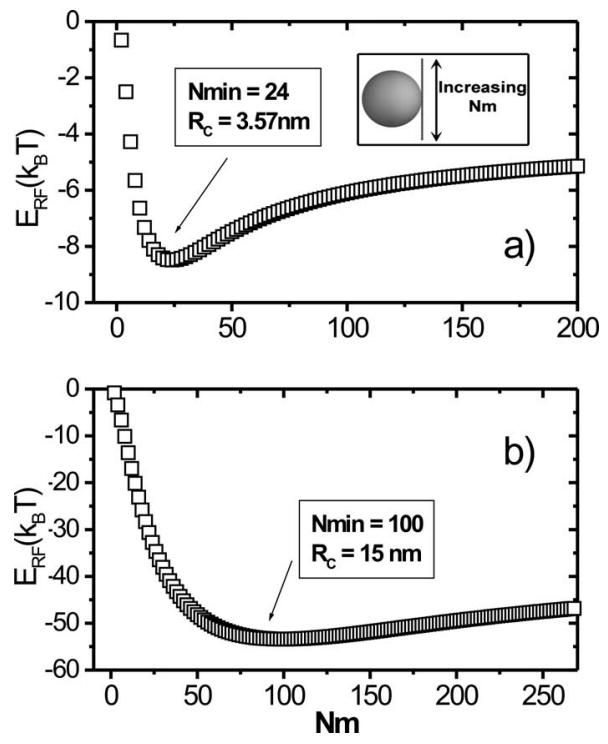

FIG. 6. Reaction field energy as a function of the PE number of monomers $\mathrm{N}_{\mathrm{m}}$. The PE is in contact with the NP as illustrated in the inset. The NP has a dielectric constant equal to 1000 . The monomer radius is fixed to $0.357 \mathrm{~nm}$ and the radius of the NP is fixed to (a) $R_{c}=3.57 \mathrm{~nm}$ and (b) $R_{c}=15 \mathrm{~nm}$. $\mathrm{N}_{\text {min }}$ corresponds to the PE number of monomers, which corresponds to an energetic minimum. It is shown that the PE length can be adjusted to promote the PE-NP interaction process. 

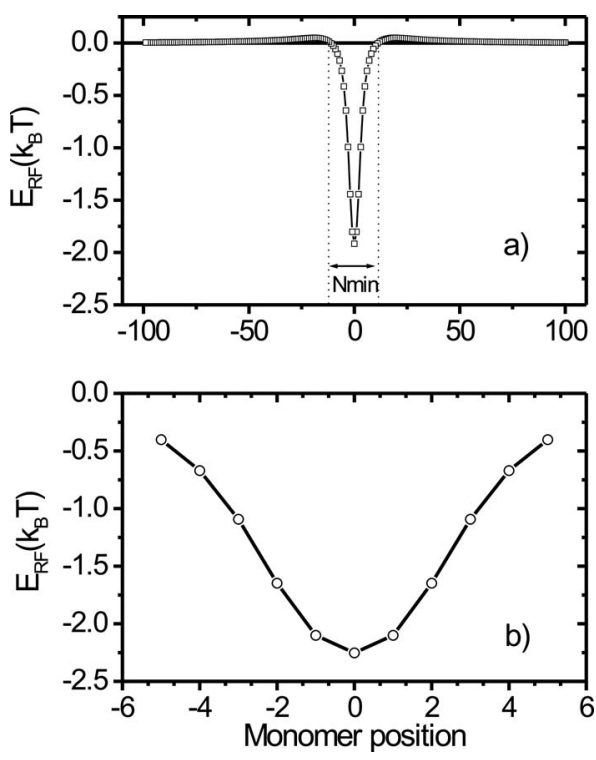

FIG. 7. (a) Reaction field energy contribution of each monomer with $\mathrm{N}_{\mathrm{m}}$ $=201$ as a function of the monomer position on the PE chain. The central monomer (at 0) is in contact with the NP. (b) Reaction field energy contribution for a PE having 11 monomers. $\mathrm{R}_{\mathrm{c}}=3.57 \mathrm{~nm}, \varepsilon_{\mathrm{c}}=1000$. Negative values are obtained by considering central monomers, whereas positive values are obtained with the rest of the monomers.

present study shows that complexation between an uncharged neutral spherical NP and a charged PE can be obtained due to the presence of image charges if the dielectric permittivity of the NP is higher than the one of the PE and the solvent. This may have a significant impact on the rational use of charged polymer for the functionalization of manufactured NPs. The increase in the NP dielectric discontinuity is also found to promote the PE adsorption. It is also found that the reaction field contribution to the PE adsorption increases by increasing both the NP radius and PE contour length. The effect of the PE length is not trivial since the intensity of the interaction between the PE and the NP can be optimized by adjusting the PE monomer number for a given NP size and dielectric constant. The dielectric discontinuity effect on the adsorption was investigated here for a rodlike PE having the same dielectric constant than the solvent. It should be very interesting to use another dielectric constant for the PE and compare the effects of the reaction field discussed here with the Coulombic interactions arising from the charges on the surface of the particles and along the polymer backbone (if oppositely charged polymers and particles are considered, which is the most common case). Future work could also concern flexible PEs to get an insight into the influence of the PE conformational changes, PE persistence length, and contour length on the effect on the reaction field energy profile. Indeed the minimum shown in Fig. 6 is expected to change if the stiffness of polymers due to the elec- trostatic and intrinsic persistence length has low values. In this case, the interactions between the charged monomers and the image charges in the NP will certainly promote the polymer adsorption at the NP surface in order to increase the attractive interactions between the charged monomers and the opposite image charges, and hence reduce the repulsive interactions between the charged monomers and image charges of the same sign in the NP.

\section{ACKNOWLEDGMENTS}

The authors are grateful to Dr. A. Vaccaro and F. Alvaltroni for their help, encouragements, and stimulating discussions. We gratefully acknowledge the financial support received from the Swiss National Science Foundation (Project No. 200020-101974/1).

${ }^{1}$ Y. Ju-Nam and J. R. Lead, Sci. Total Environ. 400, 396 (2008).

${ }^{2}$ J. Fresnais, J. F. Berret, B. Frka-Petesic, O. Sandre, and R. Perzynski, Adv. Mater. (Weinheim, Ger.) 20, 3877 (2008).

${ }^{3}$ L. Qi, A. Sehgal, J.-C. Castaing, J.-P. Chapel, J. Fresnais, J.-F. Berret, and F. Cousin, NANO 2, 879 (2008).

${ }^{4}$ S. Ulrich, M. Seijo, and S. Stoll, Curr. Opin. Colloid Interface Sci. 11, 268 (2006).

${ }^{5}$ R. R. Netz and D. Andelman, Phys. Rep. 380, 1 (2003).

${ }^{6}$ C. L. Cooper, P. L. Dubin, A. B. Kayitmazer, and S. Turksen, Curr. Opin. Colloid Interface Sci. 10, 52 (2005).

${ }^{7}$ A. V. Dobrynin and M. Rubinstein, Prog. Polym. Sci. 30, 1049 (2005).

${ }^{8}$ P. L. Dubin, S. S. The, D. W. McQuigg, C. H. Chew, and L. M. Gan, Langmuir 5, 89 (1989)

${ }^{9}$ H. Zhang, P. L. Dubin, J. Ray, G. S. Manning, C. N. Moorefield, and G. R. Newkome, J. Phys. Chem. B 103, 2347 (1999).

${ }^{10}$ X. H. Feng, P. L. Dubin, H. W. Zhang, G. F. Kirton, P. Bahadur, and J. Parotte, Macromolecules 34, 6373 (2001).

${ }^{11}$ A. B. Kayitmazer, D. Shaw, and P. L. Dubin, Macromolecules 38, 5198 (2005).

${ }^{12}$ C. L. Cooper, A. Goulding, A. B. Kayitmazer, S. Ulrich, S. Stoll, S. Turksen, S.-I. Yusa, A. Kumar, and P. L. Dubin, Biomacromolecules 7, 1025 (2006).

${ }^{13}$ F. Ganachaud, A. Elaissari, C. Pichot, A. Laayoun, and P. Cros, Langmuir 13, 701 (1997)

${ }^{14}$ A. Elaissari, Y. Chevalier, F. Ganachaud, T. Delair, and C. Pichot, Langmuir 16, 1261 (2000).

${ }^{15}$ A. Elaissari, J. P. Chauvet, M. A. Halle, O. Decavallas, C. Pichot, and P. Cros, J. Colloid Interface Sci. 202, 251 (1998).

${ }^{16} \mathrm{H}$. W. Walker and S. B. Grant, Langmuir 12, 3151 (1996).

${ }^{17}$ H. W. Walker and S. B. Grant, J. Colloid Interface Sci. 179, 552 (1996).

${ }^{18}$ G. S. Manning, J. Phys. Chem. B 107, 11485 (2003).

${ }^{19}$ A. Emelyanenko and L. Boinovich, J. Phys.: Condens. Matter 20, 494227 (2008).

${ }^{20}$ P. Linse, J. Chem. Phys. 128, 114505 (2008).

${ }^{21}$ R. R. Netz, J. Phys.: Condens. Matter 15, S239 (2003).

${ }^{22}$ C. Friedsam, H. E. Gaub, and R. R. Netz, Europhys. Lett. 72, 844 (2005).

${ }^{23}$ A. V. Dobrynin and M. Rubinstein, J. Phys. Chem. B 107, 8260 (2003).

${ }^{24}$ C.-H. Cheng and P.-Y. Lai, Phys. Rev. E 70, 061805 (2004).

${ }^{25}$ R. Messina, Phys. Rev. E 70, 051802 (2004).

${ }^{26}$ R. Messina, Phys. Rev. E 74, 049906 (2006).

${ }^{27}$ G. S. Manning, J. Chem. Phys. 51, 924 (1969).

${ }^{28}$ C. J. F. Böttcher, Theory of Electric Polarization (Elsevier, New York, 1973).

${ }^{29}$ N. Metropolis, A. W. Rosenbluth, M. N. Rosenbluth, A. H. Teller, and E. Teller, J. Chem. Phys. 21, 1087 (1953). 